\title{
AVALIAÇÃO DOS ESTADOS DE HUMOR DOS ATLETAS PARAOLÍMPICOS BRASILEIROS DO FUTEBOL DE CINCO
}

\author{
Vanessa Helena Santana Dalla Déa \\ Universidade Federal de Goiás, Goiânia, Goiás, Brasil
}

Edison Duarte

Universidade Estadual de Campinas, Campinas, São Paulo, Brasil

José Irineu Gorla

Universidade Estadual de Campinas, Campinas, São Paulo, Brasil

Humberto Luís de Deus Inácio

Universidade Federal de Goiás, Goiânia, Goiás, Brasil

\author{
Alessandra Paiva de Castro \\ Universidade Federal de São Carlos, São Carlos, São Paulo, Brasil
}

Resumo: Este estudo teve como objetivo caracterizar o estado de humor dos atletas com deficiência visual do Futebol de cinco. Participaram desta pesquisa 16 atletas, sendo 13 atletas com deficiência visual e 3 videntes (goleiros), pré-convocados pelo Comitê Paraolímpico Brasileiro para participar das Paraolimpíadas de Pequim. Para a avaliação dos estados de humor foi utilizado o intrumento Profile Mood States (POMS). Observou-se distúrbios em todos os atletas com visão normal, sendo dois no nível de tensão e um no nível de vigor. Nos atletas com deficiência visual apenas 38,5\% apresentou alterações nos estados de humor. Os atletas com melhores índices no Estado de Humor foram selecionados para representar o Brasil nas Paraolimpíadas de Pequim.

Palavras-chave: Deficiência. Futebol. Estado Psicológico.

\section{Introdução}

Inicialmente a Educação Física foi fundamentada por princípios oriundos das ciências biológicas (Daolio, 1998). Com esta ênfase, as atividades executadas nas aulas de educação física têm como objetivo desenvolver exclusivamente a aptidão física do aluno. A partir da busca por alternativas, iniciou-se um processo de crítica ao predomínio biológico e admitiu-se uma Educação Física pluridimensional, especialmente nos aspectos social e cultural. A Educação Física é uma atividade não só física, mas também psico-social. Já nas décadas de 1920 e 1930, Coleman Griffith deu grandes contribuições apresentando aspectos psicológicos relacionados com o esporte. Este autor é considerado o pai da psicologia de Treinamento (Singer, 1977).

Pensar a Prática, Goiânia, v. 14, n. 2, p. 1-10, maio/ago. 2011 
De modo mais genérico, e pela especificidade deste estudo, vamos nos referir daqui para frente não à educação física, mas sim à atividade física, entendida como um conteúdo da primeira. ${ }^{1}$

Já há algum tempo, a relação existente entre a atividade física e a expressão de emoções vem sendo colocada em debate. Miranda (1998) relata que qualquer atividade física nunca é puramente física, sempre implica em uma simultaneidade psíquica que nem sempre é levada em consideração por praticantes ou pelos professores envolvidos. A mesma autora relata a forte relação entre atividade física, emoções e comportamento, relatando que um dos fatores mais explícitos nas atividades físicas e esportes de um modo geral, é o efeito emocional sobre o comportamento da pessoa diante de uma tarefa qualquer. Há uma clássica discussão a respeito das emoções no que se refere ao efeito perturbador e desorganizador sobre o comportamento.

Gazzaniga e Heatherton (2005) relatam que o humor ou estado de ânimo variam com as vivências e exigências proporcionadas pelo meio externo, podendo ser definido como o tônus afetivo do indivíduo, Segundo os autores o estado de humor influencia a forma como o indivíduo irá perceber o que está a sua volta, aumentando ou reduzindo o impacto destes acontecimentos e determinando como irá agir.

Weineck (1990) relata que para o bom desempenho de atletas é importante o bem estar (aspectos determinantes relacionados com fatores fisiológicos, sociais e psicológicos, presentes na unicidade esportiva). Jones e Hardy (1990) estabelecem que a eficiência dos atletas esta envolvida com as variáveis cognitivas e psicológicas do indivíduo, o que significa a associação de fatores biomecânicos, fisiológicos e principalmente psicológicos para o sucesso.

Alterações psicológicas que influenciam no rendimento esportivo são constantemente observadas em atletas e podem surgir por inúmeros motivos. As grandes exigências físicas e psicológicas dos treinos e competições, a incerteza da vitória, o medo da derrota, a insegurança quanto as suas capacidades e habilidades, são alguns dos muitos fatores que podem propiciar o desequilíbrio emocional no atleta (Rohlfs et al, 2004).

Vieira et al (2008) relatam que o estado de humor é um fator que interfere diretamente no desempenho destas equipes de alto rendimento. Os autores dizem ainda que o esporte apresenta-se como um dos ambientes que podem influenciar na estrutura da personalidade do atleta alterando os estados de humor deste. Quando o estado de humor se desestabiliza o atleta apresenta dificuldades em viver e funcionar no âmbito social, podendo fugir das normas, regras e comportamentos adequados no esporte em questão podendo prejudicar sua equipe

Um dos instrumentos mais utilizados para mensurar o bem estar psicológico no meio esportivo atualmente é o Profile Mood States (POMS), utilizado para avaliar estados de humor em diversos estudos (Polman, Kaiseler e Borkoles, 2007; Audette et al, 2006; Berlin, Kop e Deuster, 2006; Scott, McNaughton e Polman, 2006; Galantino et

1 Importante debate está estabelecido no campo da educação física sobre as diferenças conceituais entre as expressões 'exercíco físico', 'atividade física' e 'prática corporal'. Neste momento, optamos pela expressão 'atividade física' por sua aceitação mais ampla na área esportiva, mas indicando que 'prática corporal' tende a representar melhor a perspectiva de uma educação física mais avançada.

Pensar a Prática, Goiânia, v. 14, n. 2, p. 1-10, maio/ago. 2011 
al, 2005; Bartholomew, Morrison e Ciccolo, 2005; Ohta et al, 2005; Esposito et al, 2005; Annesi, 2005; Rietjens et al, 2005).

Quando se trata de um atleta com deficiência outros fatores se somam aos convencionais como o preconceito e as barreiras para acessibilidade. A preocupação e pesquisa com o acompanhamento psicológico dos atletas paraolímpicos pode ser observada nos estudos de Samulski e Noce (2002), Samulski (2001) e Samulski (2003).

\section{Objetivo}

Este estudo teve como objetivo caracterizar o estado de humor dos atletas do Futebol de cinco momentos antes da seleção dos atletas que representaram o Brasil nas olimpíadas de Pequim. Esta modalidade é praticada por pessoas com deficiência visual, sendo que apenas os goleiros são videntes.

\section{Método}

Esta pesquisa foi solicitada e autorizada pelo Comitê Paraolímpico Brasileiro e Comitê Científico Paraolímpico. Após a pesquisa os dados foram apresentados à Comissão Técnica e utilizados como subsídio para treinamento e seleção dos atletas.

\section{Sujeitos}

Participaram desta pesquisa 16 atletas convocados pelo Comitê Paraolímpico Brasileiro para treinamento e seleção dos 13 atletas que representaram o Brasil na equipe do Futebol de cinco nas Paraolimpíadas de Pequim sendo 13 atletas com deficiência visual e 3 atletas videntes que ocupam a posição de goleiro.

\section{Medida}

O Profile Mood States (POMS) é um instrumento inicialmente desenvolvido para avaliação psiquiátrica publicado em sua forma original por McNair, Loor e Droppleman (1971). Posteriormente foi validado para aplicação esportiva, e adaptado para a língua portuguesa em versão reduzida por Peluso (2003).

Para a utilização do POMS no âmbito esportivo foi criado um modelo de estrutura mental que visa caracterizar o desempenho ótimo que se tornou um conceito clássico na Psicologia do Desporto denominado Perfil Iceberg (Bell e Howe, 1986; Morgan, 1980,1985).

Este questionário apresenta uma forma de rápida e fácil aplicação e apresenta informações pertinentes para a preparação de atletas. Segundo Viana, Almeida e Santos (2001) permite avaliar precisamente 6 estados de humor:

1. Tensão-ansiedade: indica aumento de tensão músculo esquelética e preocupação.

2. Depressão-melancolia: representa um estado emocional de tristeza, desanimo, infelicidade e solidão.

3. Hostilidade-ira: estado de humor de antipatia em relação aos outros.

Pensar a Prática, Goiânia, v. 14, n. 2, p. 1-10, maio/ago. 2011 
4. Vigor-atividade: relaciona-se com estado de energia e vigor físico e psicológico.

5. Fadiga-inércia: representa um estado de cansaço e de baixa energia.

6. Confusão-desorientação: caracteriza-se por um estado de confusão e baixa lucidez.

As pontuações de cada item obedecem a escala de Likert de 5 pontos: "nada" (0), "um pouco" (1), "mais ou menos" (2), "bastante" (3) e "extremamente" (4). Para esta avaliação solicitou-se ao avaliado que dissesse como tem se sentido nos últimos sete dias considerando o dia da avaliação (Viana, Almeida e Santos, 2001).

O POMS não precisou ser adaptado para pesquisa com pessoas com deficiência visual, pois se mostrou de fácil entendimento na realização de pesquisa piloto. $O$ instrumento foi lido pelo mesmo avaliador para todos os atletas que verbalmente sinalizavam a alternativa correta para seu estado de humor.

\section{Resultados}

Para análise dos gráficos é importante saber que o valor de percentil 50 foi estabelecidos em estudos anteriores como a média dos valores da população não-atleta e os valores 40 e 60 são considerados desvios padrões dessa população. Para uma população de atletas espera-se como ideal um valor maior do que de 60 ou maior para o fator vigor e menor que 40 para os fatores negativos (tensão, depressão, hostilidade, fadiga e confusão).

A Tabela 1 apresenta os valores percentuais encontrados em cada um dos atletas. Sendo que não são deficientes visuais os atletas $\mathrm{B}, \mathrm{M}$ e $\mathrm{N}$, isto é ocupam a posição de goleiros.

\begin{tabular}{|c|c|c|c|c|c|c|}
\hline Atletas & Tensão & Depressão & Hostilidade & Vigor & Fadiga & Confusão \\
\hline A. & 21 & 2 & 0 & 100 & 21 & 0 \\
\hline B. & $* 42$ & 0 & 0 & 100 & 0 & 0 \\
\hline C. & 21 & 0 & 4 & 87 & 4 & 4 \\
\hline D. & $* 46$ & 10 & 6 & 83 & 21 & 6 \\
\hline E. & 25 & 2 & 0 & 71 & 0 & 4 \\
\hline F. & 17 & 0 & 4 & $* 0$ & 0 & 21 \\
\hline G. & 17 & 4 & 8 & 62 & 8 & 8 \\
\hline H. & $* 42$ & 12 & 6 & 71 & 21 & 21 \\
\hline I. & 25 & 6 & 0 & 92 & 0 & 17 \\
\hline $\mathbf{J}$. & 29 & 2 & 8 & 96 & 8 & 8 \\
\hline
\end{tabular}

Pensar a Prática, Goiânia, v. 14, n. 2, p. 1-10, maio/ago. 2011 


\begin{tabular}{|c|c|c|c|c|c|c|}
\hline & & & & & & \\
\hline K. & 21 & 0 & 0 & 75 & 0 & 6 \\
\hline L. & $* 63$ & 4 & 37 & 76 & 25 & 8 \\
\hline M. & 34 & 4 & 4 & $* 58$ & 8 & 17 \\
\hline N. & $* 62$ & 18 & 25 & 62 & 8 & 9 \\
\hline O. & 12 & 0 & 4 & $* 42$ & 12 & 4 \\
\hline P. & $* 42$ & 6 & 16 & $* 12$ & 37 & 37 \\
\hline
\end{tabular}

*Valores fora do padrão esperado para atletas.

Alterações foram detectadas em 56,25\% dos atletas, isto é 9 dos 16 atletas.

Os resultados encontrados para os Estados de Humor, para cada um dos atletas analisados, pode ser melhor observado na Figura 1.

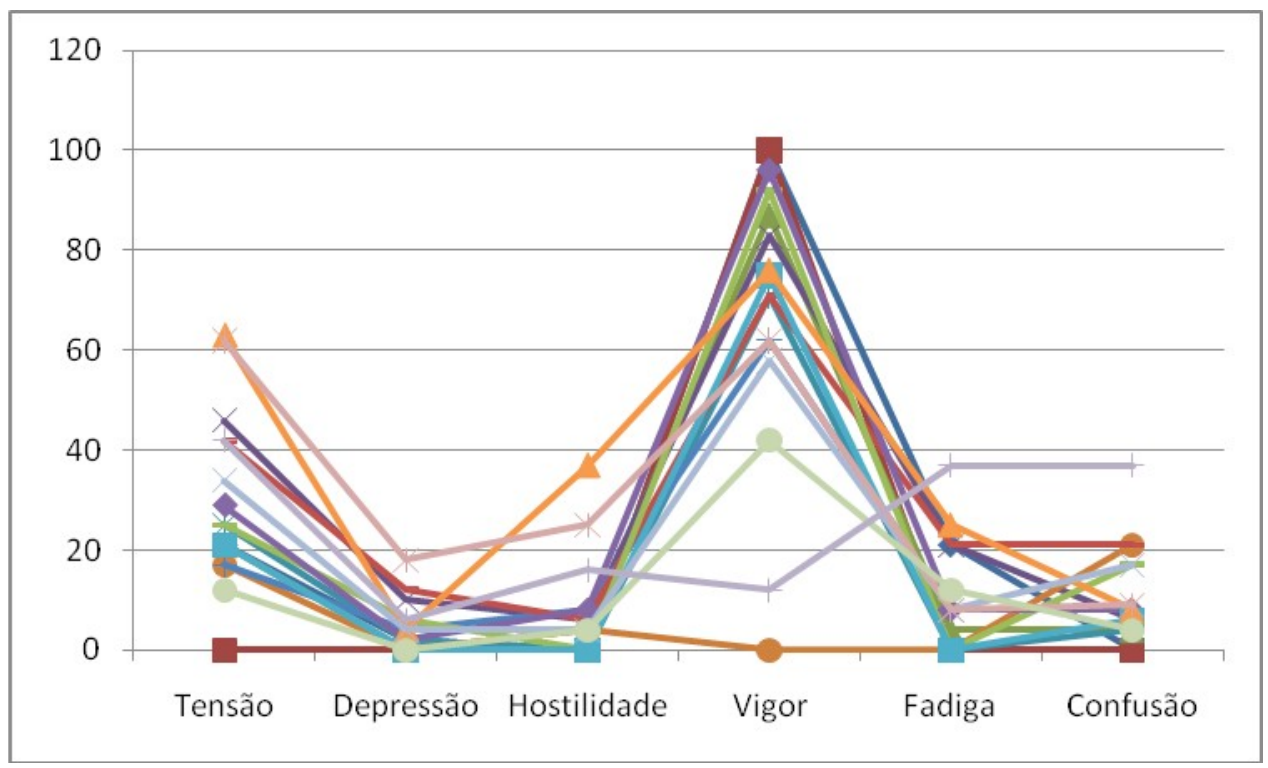

FIGURA 1: Perfil do estado de humor dos atletas do futebol de 5.

\section{Discussão}

Conscientes que o bom rendimento físico depende, entre outros fatores, do estado psicológico do atleta, os escores resultantes desta pesquisa proporcionam uma visualização deste estado e a predisposição dos atletas para a aquisição de capacidade e habilidade física.

Pensar a Prática, Goiânia, v. 14, n. 2, p. 1-10, maio/ago. 2011 
Verificou-se que $62,5 \%$ dos atletas apresentarem alterações em pelo menos um dos seis estados de humor avaliados, sendo que os três atletas com visão normal que ocupam a posição de goleiro estão inclusos. Se considerados apenas os atletas com deficiência visual esta porcentagem cai para apenas 38,5\% com alterações no estado de humor.

Ao estudar uma equipe de voleibol de alto rendimento Vieira et al (2008) encontraram como valores médios dos estados de humor os seguintes: Tensão de 42,0, depressão em 41,25, Raiva de 45,67, Vigor de 60,50, Fadiga de 32,75 e confusão de 37,08. Os autores mostram que na fase de treinamento o estado de humor apresenta-se mais alterado que na fase de competição.

Apesar deste estudo com atletas paraolímpicos ter sido na fase de treinamento, e aumentando ainda mais a dificuldade, de seleção dos atletas, os índices encontrados foram significativamente menores. As médias encontradas foram: tensão 31,8, depressão 4,3, raiva ou hostilidade 7,62, vigor 67,93, fadiga 10,81 e confusão 10,62.

Comparando com o estudo de Vieira et al (2008) pode-se concluir que os níveis do estado de humor dos atletas paraolímpicos é mais adequado que dos atletas de voleibol. Pode-se verificar que o nível de depressão, hostilidade, fadiga e confusão enquadram-se no ideal para atletas. Estes dados, entre outros, podem justificar os resultados obtidos pela equipe atualmente: tri-campeões paraolímpicos e campeões mundiais.

As alterações no nível de vigor foram encontradas em apenas quatro dos dezesseis avaliados, sendo que três destes atletas não foram selecionados pela comissão técnica da equipe para representar o Brasil nas Paraolimpíadas de Pequim.

$\mathrm{O}$ estado de humor onde detectou-se maior número de alterações indesejadas foi no nível de tensão (37,5\% dos atletas avaliados), este fato pode ser justificado pelo fato de que a coleta de dados foi realizada próxima do período de eliminação dos atletas que não fariam parte da equipe principal.

É importante relatar que foram feitos pelo Comitê Científico muitos outros testes físicos e psicológicos, além do apresentado neste estudo, para auxiliar a comissão técnica da seleção paraolímpica brasileira do futebol de cinco à selecionar os atletas para as Paraolimpíadas de Pequim. Os resultados deste estudo mostram que os atletas não convocados pelo Comitê Paraolímpico Brasileiro apresentaram as maiores alterações no estado de humor.

\section{Conclusão}

Na equipe de futebol de 5 em fase de treinamento para as Olimpíadas de Pequim foi possível verificar nível adequado nos estados de humor: depressão, hostilidade, fadiga e confusão. Observou-se alterações em todos os atletas com visão normal, sendo dois no nível de tensão e um no nível de vigor. Nos atletas com deficiência visual apenas $38,5 \%$ apresentou alterações.

Pensar a Prática, Goiânia, v. 14, n. 2, p. 1-10, maio/ago. 2011 


\section{Referências}

ANNESI, J.J. Changes in depressed mood associated with 10 weeks of moderate cardiovascular exercise in formerly sedentary adults. Psychol Rep; 96(3 Pt 1):855-62, 2005.

AUDETTE, J.F.; JIN, Y.S.; NEWCOMER, R.; STEIN, L.; DUNCAN, G.; FRONTERA, W.R. Tai Chi versus brisk walking in elderly women. Ageing;35(4):388-93, 2006.

BARTHOLOMEW, J.B.; MORRISON, D.; CICCOLO, J.T. Effects of acute exercise on mood and well-being in patients with major depressive disorder. Med Sci Sports Exerc;37(12):2032-7, 2005 Dec.

BELL, G. J.; HOWE, B. L. Mood state profiles and motivations of triathletes. Journal of Sport Behavior, 11, 66-77, 1986.

BERLIN, A.A.; KOP, W.J.; DEUSTER, P.A. Depressive mood symptoms and fatigue after exercise withdrawal: the potential role of decreased fitness. Psychosom Med; 68(2):224-30, 2006.

DAOLIO, J. Educação Física e Cultura. Corpoconsciência, Santo André, n.1, $1^{\text { }}$. semestre, 1998.

ESPOSITO, J.G.; THOMAS, S.G.; KINGDON, L.; EZZAT, S. Anabolic growth hormone action improves submaximal measures of physical performance in patients with HIV-associated wasting. Am J Physiol Endocrinol Metab; 289(3):E494-503, 2005.

GALANTINO, M.L.; SHEPARD, K.; KRAFFT, L.; LAPERRIERE, A.; DUCETTE, J.; SORBELLO, A.; BARNISH, M.; CONDOLUCI, D.; FARRAR, J.T. The effect of group aerobic exercise and t'ai chi on functional outcomes and quality of life for persons living with acquired immunodeficiency syndrome. J Altern Complement Med; 11(6):1085-92, 2005 Dec

GAZZANIGA, M.S.; HEATHERTON, T.F. Ciência psicológica: mente, cérebro e comportamento. Porto Alegre: Artmed; 2005.

JONES, J. G.; HARDY, L. Stress and performance in sport. Chichester: Willey and Sounds, 1990.

MIRANDA, R. Atividade física e emoção. In Simpósio Mineiro de Psicologia do Esporte Juiz de Fora: Universidade Federal de Juiz de Fora, Faculdade de Educação Física e Desportos: EDUFJF, 1998. 
MCNAIR, D. M., LOOR, M., E DROPPLEMAN, L. F. Manual for the Profile of Mood States. San Diego, California: EdITS/Educational and Industrial Testing Service, 1971.

MORGAN, W. P. Test of Champions: the iceberg profile. Psychology Today, 14, 9299, 101-108, 1980.

MORGAN, W. P. Affective beneficence of vigorous physical activity. Medicine and Science in Sports and Exercise, 17, 94-100, 1985.

OLIVEIRA, S.R.S.; SERASSUELO JUNIOR, H.; SIMÕES, A. C. Caracterização e identificação dos estados de humor de atletas do judô. In: 4o. Congresso LatinoAmericano de Educação Física - FACIS - UNIMEP, 2006.

OHTA, M.; HIRAI, N.; ONO, Y.; OHARA, M.; SAITO, S.; HORIGUCHI, S.; WATANABE, M.; TOKASHIKI, A.; KAWAI, A.; ANDOU, T.; SHIOJI, I.; NOGUCHI, T.; MORIZUKA, M.; SUZUKI, M.; IMANISHI, A.; TAKEDA, N.; MACHIDA, K. Clinical biochemical evaluation of central fatigue with 24-hour continuous exercise. Rinsho Byori; 53(9):802-9, 2005.

PELUSO, M.A.M. Alterações de Humor associadas a atividade física intensa. Faculdade de Medicina. São Paulo: Faculdade de Medicina da Universidade de São Paulo, tese de Doutorado, 2003.

POLMAN, R.; KAISELER, M.; BORKOLES, E. Effect of a single bout of exercise on the mood of pregnant women. J Sports Med Phys Fitness; 47(1):103-11, 2007.

RIETJENS, G.J.; KUIPERS, H.; ADAM, J.J.; SARIS, W.H.; VAN BREDA, E.; VAN HAMONT, D.; KEIZER, H.A. Physiological, biochemical and psychological markers of strenuous training-induced fatigue. Int J Sports Med;26(1):16-26, 2005.

ROHLFS, I.C.P.M.; CARVALHO, T. DE; ROTTA, T.M.; KREBS, R.J. Aplicação de instrumentos de avaliação de estados de humor na detecção da síndrome do excesso de treinamento. Revista Brasileira de Medicina de Esporte. Vol. 10, no. 2, mar/abr, 2004

SCOTT, J.P.; MCNAUGHTON, L.R.; POLMAN, R.C. Effects of sleep deprivation and exercise on cognitive, motor performance and mood. Physiol Behav; 87(2):396-408, 2006.

SINGER, R.N. Psicologia dos esportes: mitos e verdades. São Paulo: Harper \& Row do Brasil, 1977.

WEINECK, J. Treinamento ideal. São Paulo: Manole, 9ª.ed., 1999.

Pensar a Prática, Goiânia, v. 14, n. 2, p. 1-10, maio/ago. 2011 
VIANA, M. F.; ALMEIDA, P. L.; SANTOS, R. C. Adaptação portuguesa da versão reduzida do Perfil de Estados de Humor - POMS. Análise Psicológica, 1 (XIX): 77-92, 2001

VIEIRA, L.F; FERNANDES, S.L.; VIEIRA, J.L.L.; VISSOCI, J.R.N. Estado de humor e desempenho motor: um estudo com atletas de voleibol de alto rendimento. Revista Brasileira de Cineantropometria \& Desempenho Humano, 10 (1):62-68, 2008.

\title{
ASSESSMENT OF MOOD STATES OF BRAZILIAN PARALYMPIANS FOOTBALL FIVE
}

\begin{abstract}
The aim of this study was to characterize the mood of visually impaired athletes in soccer five. 16 athletes participated in this study, 13 athletes with visual impairment and three seers (goalkeepers), pre-selected by the Brazilian Paralympic Committee to attend the Paralympics in Beijing. For the evaluation of mood states the Profile Mood States (POMS) was used. Disorders was observed in all athletes with normal vision, two with voltage level and one with a force level. In visually impaired athletes only $38.5 \%$ showed changes in mood states. Athletes with better mood state were selected to represent Brazil at the Paralympics in Beijing.
\end{abstract}

Keywords: Disability. Football. Psychological State.

\section{EVALUACIÓN DEL ESTADO DE ÁNIMO DE LOS ATLETAS PARALÍMPICOS BRASILEÑOS DEL FÚTBOL DE CINCO}

Resumen: Este estudio tuvo como objetivo caracterizar el estado de ánimo de atletas del fútbol de cinco. Esta modalidad es practicada por personas con discapacidad visual, donde sólo el portero es vidente. Se analizaron los 16 atletas, 13 atletas con deficiencias visuales y videntes tres atletas que ocupan la posición de portero, todos convocados por el Comité Paralímpico Brasileño para la entrenamiento y selección de los 13 atletas que representaran a Brasil en el equipo de fútbol de cinco en los Juegos Paralímpicos Beijing. Para la evaluación del estado de ánimo se utilizó el instrumento Profile Mood States (POMS). En el equipo de fútbol de cinco en la fase de entrenamiento para los Juegos Olímpicos de Beijing fue posible verificar el nivel adecuado del estado de ánimo: depresión, hostilidad, fatiga y confusión. Se observó trastornos en todos los atletas con visión normal, siendo dos en el nivel de tensión y un en el nivel de fuerza. En los atletas con discapacidad visual sólo el 38,5\% mostró cambios en los estados de ánimo. Los atletas con mejor estado de ánimo fueron seleccionados para representar a Brasil en los Juegos Paralímpicos de Beijing.

Palabras clave: Discapacidad, el fútbol, el estado psicológico.

Pensar a Prática, Goiânia, v. 14, n. 2, p. 1-10, maio/ago. 2011 
10.5216/rpp.v14i2.14970

Endereço para correspodência:

vanessasantana@ig.com.br

Vanessa Helena Santana Dalla Déa

Universidade Federal de Goiás

Faculdade de Educação Física

Campus Samambaia, Caixa Postal 131

CEP: 74001-970 Goiânia - Goiás - Brasil

Pensar a Prática, Goiânia, v. 14, n. 2, p. 1-10, maio/ago. 2011 